\title{
New Subclasses of Multivalent Analytic Functions Associated with a Linear Operator
}

\author{
Ding-Gong Yang ${ }^{1}$ and Jin-Lin Liu ${ }^{2}$ \\ ${ }^{1}$ School of Mathematical Sciences, Soochow University, Suzhou, Jiangsu 215006, China \\ ${ }^{2}$ Department of Mathematics, Yangzhou University, Yangzhou, Jiangsu 225002, China \\ Correspondence should be addressed to Jin-Lin Liu; jlliu@yzu.edu.cn
}

Received 14 August 2013; Accepted 29 October 2013

Academic Editor: Ngai-Ching Wong

Copyright (C) 2013 D.-G. Yang and J.-L. Liu. This is an open access article distributed under the Creative Commons Attribution License, which permits unrestricted use, distribution, and reproduction in any medium, provided the original work is properly cited.

Making use of a linear operator, which is defined here by means of the Hadamard product (or convolution), we consider two subclasses $F_{p, n}(a, c, \lambda, A, B)$ and $G_{p, n}(a, c, \lambda, A, B)$ of multivalent analytic functions with negative coefficients in the open unit disk. Some modified Hadamard products, integral transforms, and the partial sums of functions belonging to these classes are studied.

\section{Introduction}

Let $A_{p, n}$ denote the class of functions of the form

$$
f(z)=z^{p}+\sum_{k=p+n}^{\infty} a_{k} z^{k}, \quad(p, n \in N=\{1,2,3, \ldots\}),
$$

which are analytic in the open unit $\operatorname{disk} U=\{z:|z|<1\}$.

For functions $f(z)$ and $g(z)$ analytic in $U$, we say that $f(z)$ is subordinate to $g(z)$ in $U$, written $f(z) \prec g(z)(z \in U)$, if there exists an analytic function $w(z)$ in $U$ such that

$$
|w(z)| \leq|z|, \quad f(z)=g(w(z)), \quad(z \in U) .
$$

Furthermore, if the function $g(z)$ is univalent in $U$, then

$$
\begin{gathered}
f(z)<g(z) \quad(z \in U) \Longleftrightarrow f(0)=g(0), \\
f(U) \subseteq g(U) .
\end{gathered}
$$

In terms of the Pochhammer symbol $(b)_{n}$ given by $(b)_{n}=$ $b(b+1) \cdots(b+n-1)(n \in N)$, we define the function $\varphi_{p, n}(a, c ; z)$ by

$$
\begin{array}{r}
\varphi_{p, n}(a, c ; z)=z^{p}+\sum_{k=p+n}^{\infty} \frac{(a)_{k-p}}{(c)_{k-p}} z^{k} \\
(z \in U ; c \notin\{0,-1,-2, \ldots\}) .
\end{array}
$$

Corresponding to $\varphi_{p, n}(a, c ; z)$, we consider here a linear operator $L_{p, n}(a, c)$ on $A_{p, n}$ by the following usual Hadamard product (or convolution):

$$
\begin{aligned}
L_{p, n}(a, c) f(z) & =\varphi_{p, n}(a, c ; z) * f(z) \\
& =z^{p}+\sum_{k=p+n}^{\infty} \frac{(a)_{k-p}}{(c)_{k-p}} a_{k} z^{k},
\end{aligned}
$$

for $f(z)$ given by (1). For $p=n=1, L_{1,1}(a, c)$ on $A_{1,1}$ was first defined by Carlson and Shaffer [1]. Its differential-integral representation can be found in [2]. We remark in passing that a much more general convolution operator than the operator $L_{p, 1}(a, c)$ was introduced by Dziok and Srivastava [3].

Let $T_{p, n}$ denote the subclass of $A_{p, n}$ consisting of functions of the form

$$
f(z)=z^{p}-\sum_{k=p+n}^{\infty} a_{k} z^{k}, \quad\left(a_{k} \geq 0 ; p, n \in N\right) .
$$

We now consider the following two subclasses of the class $T_{p, n}$. 
Definition 1. A function $f(z) \in T_{p, n}$ is said to be in the class $F_{p, n}(a, c, \lambda, A, B)$ if and only if

$$
\begin{aligned}
(1-\lambda) & \frac{L_{p, n}(a, c) f(z)}{z^{p}}+\lambda \frac{\left(L_{p, n}(a, c) f(z)\right)^{\prime}}{p z^{p-1}} \\
& \prec \frac{1+A z}{1+B z} \quad(z \in U),
\end{aligned}
$$

where

$$
a>0, \quad c>0, \quad \lambda \geq 0, \quad-1 \leq B \leq 0, \quad B<A \leq 1 .
$$

Definition 2. A function $f(z) \in T_{p, n}$ is said to be in the class $G_{p, n}(a, c, \lambda, A, B)$ if and only if

$$
\frac{z f^{\prime}(z)}{p} \in F_{p, n}(a, c, \lambda, A, B) .
$$

For functions $f_{j}(z) \in T_{p, n}$ given by

$$
f_{j}(z)=z^{p}-\sum_{k=p+n}^{\infty} a_{k, j} z^{k}, \quad\left(a_{k, j} \geq 0 ; j=1,2\right),
$$

we denote by $\left(f_{1} \cdot f_{2}\right)(z)$ the modified Hadamard (or quasiHadamard) product of $f_{1}(z)$ and $f_{2}(z)$; that is,

$$
\left(f_{1} \cdot f_{2}\right)(z)=z^{p}-\sum_{k=p+n}^{\infty} a_{k, 1} a_{k, 2} z^{k}=\left(f_{2} \cdot f_{1}\right)(z) .
$$

The class

$$
\begin{array}{r}
F_{p, n}\left(1,1, \lambda, 1-\frac{2 \alpha}{p},-1\right)=F_{p}(n, \lambda, \alpha), \\
(\lambda \geq 0 ; 0 \leq \alpha<p)
\end{array}
$$

with $n=1$ was introduced and studied earlier by Lee et al. [4] (and was further investigated by Aouf and Darwish [5], Aouf et al. [6], and Yaguchi et al. [7]). The class

$$
\begin{array}{r}
G_{p, n}\left(1,1, \lambda, 1-\frac{2 \alpha}{p},-1\right)=G_{p}(n, \lambda, \alpha), \\
(\lambda \geq 0 ; 0 \leq \alpha<p)
\end{array}
$$

with $n=1$ was studied by Aouf [8] and Aouf et al. [6]. Recently, Aouf [9] investigated the modified Hadamard products of several functions in the classes $F_{p}(n, \lambda, \alpha)$ and $G_{p}(n, \lambda, \alpha)$ for $n \in N$.

In the present paper, we prove a number of theorems involving the modified Hadamard products, integral transforms, and the partial sums of functions in the classes $F_{p, n}(a, c, \lambda, A, B)$ and $G_{p, n}(a, c, \lambda, A, B)$. Some of our results are generalizations of the corresponding results in [4-9].

In proving our main results, we need the following lemmas.

Lemma 3 (see $[10,11]$ ). A function $f(z) \in T_{p, n}$ defined by (6) is in the class $F_{p, n}(a, c, \lambda, A, B)$ if and only if

$$
\sum_{k=p+n}^{\infty}(p+(k-p) \lambda) \frac{(a)_{k-p}}{(c)_{k-p}} a_{k} \leq p \frac{A-B}{1-B}
$$

Lemma 4 (see $[10,11]$ ). A function $f(z) \in T_{p, n}$ defined by (6) is in the class $G_{p, n}(a, c, \lambda, A, B)$ if and only if

$$
\sum_{k=p+n}^{\infty} k(p+(k-p) \lambda) \frac{(a)_{k-p}}{(c)_{k-p}} a_{k} \leq p^{2} \frac{A-B}{1-B} .
$$

Making use of Lemmas 3 and 4, we can show the following two results.

Corollary 5. Let

$$
\begin{gathered}
0<a_{1} \leq a_{0}, \quad 0<c_{0} \leq c_{1}, \quad 0 \leq \lambda_{1} \leq \lambda_{0}, \\
-1 \leq B_{1} \leq B_{0}<A_{0} \leq A_{1} \leq 1, \quad B_{0} \leq 0 .
\end{gathered}
$$

Then

$$
\begin{gathered}
F_{p, n}\left(a_{0}, c_{0}, \lambda_{0}, A_{0}, B_{0}\right) \subseteq F_{p, n}\left(a_{1}, c_{1}, \lambda_{1}, A_{1}, B_{1}\right), \\
G_{p, n}\left(a_{0}, c_{0}, \lambda_{0}, A_{0}, B_{0}\right) \subseteq G_{p, n}\left(a_{1}, c_{1}, \lambda_{1}, A_{1}, B_{1}\right) .
\end{gathered}
$$

Corollary 6. Let $f(z) \in T_{p, n}$. Then $f(z) \in F_{p, n}\left(a_{0}, c_{0}, \lambda_{0}, A\right.$, B) $\left(G_{p, n}\left(a_{0}, c_{0}, \lambda_{0}, A, B\right)\right)$ if and only if $(f * g)(z) \in F_{p, n}\left(a_{1}\right.$, $\left.c_{1}, \lambda_{1}, A, B\right)\left(G_{p, n}\left(a_{1}, c_{1}, \lambda_{1}, A, B\right)\right)$, where $\lambda_{0} \geq 0, \lambda_{1} \geq$ $0, a_{j}>0$ and $c_{j}>0(j=0,1)$, and

$$
g(z)=z^{p}+\sum_{k=p+n}^{\infty} \frac{\left(p+(k-p) \lambda_{0}\right)\left(a_{0}\right)_{k-p}\left(c_{1}\right)_{k-p}}{\left(p+(k-p) \lambda_{1}\right)\left(a_{1}\right)_{k-p}\left(c_{0}\right)_{k-p}} z^{k} .
$$

If we let

$$
a=c=1, \quad A=1-\frac{2 \alpha}{p}, \quad 0 \leq \alpha<p, \quad B=-1,
$$

then Lemmas 3 and 4 reduce to the following result.

Corollary 7. Let $f(z) \in T_{p, n}$ be defined by (6). Then

(i) $f(z)$ is in the class $F_{p}(n, \lambda, \alpha)$ if and only if

$$
\sum_{k=p+n}^{\infty}(p+(k-p) \lambda) a_{k} \leq p-\alpha
$$

(ii) $f(z)$ is in the class $G_{p}(n, \lambda, \alpha)$ if and only if

$$
\sum_{k=p+n}^{\infty} k(p+(k-p) \lambda) a_{k} \leq p(p-\alpha)
$$

\section{Modified Hadamard Products}

Hereafter in this paper we assume that (8) is satisfied:

$$
\begin{array}{r}
0 \leq \alpha<p, \quad 0 \leq \alpha_{j}<p, \quad-1 \leq B_{j} \leq 0, \\
B_{j}<A_{j} \leq 1, \quad(j=1,2, \ldots, m) .
\end{array}
$$

Theorem 8. Let $f_{j}(z) \in F_{p, n}\left(a, c, \lambda, A_{j}, B_{j}\right)(j=1,2, \ldots, m)$ and $a \geq c>0$. Then

$$
\left(f_{1} \cdot f_{2} \cdot \ldots \cdot f_{m}\right)(z) \in F_{p, n}(a, c, \lambda, A(B), B),
$$


where $m \geq 2$ and

$$
A(B)=B+(1-B)\left(\frac{p(c)_{n}}{(p+n \lambda)(a)_{n}}\right)^{m-1} \prod_{j=1}^{m}\left(\frac{A_{j}-B_{j}}{1-B_{j}}\right) .
$$

The result is sharp; that is, $A(B)$ cannot be decreased for each $B \in[-1,0]$.

Proof. By (24) we have $B<A(B) \leq 1$ for $a \geq c>0$. Let

$$
\begin{array}{r}
f_{j}(z)=z^{p}-\sum_{k=p+n}^{\infty} a_{k, j} z^{k} \in F_{p, n}\left(a, c, \lambda, A_{j}, B_{j}\right) \\
\left(a_{k, j} \geq 0 ; j=1,2, \ldots, m\right) .
\end{array}
$$

Then Lemma 3 gives

$$
\begin{aligned}
& \left(\frac{1-B_{j}}{A_{j}-B_{j}}\right) \sum_{k=p+n}^{\infty} \frac{(p+(k-p) \lambda)(a)_{k-p}}{p(c)_{k-p}} a_{k, j} \\
& \leq 1 \quad(j=1,2, \ldots, m),
\end{aligned}
$$

and hence

$$
\begin{aligned}
\prod_{j=1}^{m}\left(\frac{1-B_{j}}{A_{j}-B_{j}}\right) \\
\quad \times \sum_{k=p+n}^{\infty}\left(\frac{(p+(k-p) \lambda)(a)_{k-p}}{p(c)_{k-p}}\right)^{m} a_{k, 1} a_{k, 2} \cdots a_{k, m} \\
\leq \prod_{j=1}^{m}\left\{\left(\frac{1-B_{j}}{A_{j}-B_{j}}\right) \sum_{k=p+n}^{\infty} \frac{(p+(k-p) \lambda)(a)_{k-p}}{p(c)_{k-p}} a_{k, j}\right\}
\end{aligned}
$$$$
\leq 1
$$

Also, using Lemma 3,

$$
\begin{aligned}
h(z) & =\left(f_{1} \cdot f_{2} \cdot \ldots \cdot f_{m}\right)(z) \\
& =z^{p}-\sum_{k=p+n}^{\infty} a_{k, 1} a_{k, 2} \cdots a_{k, m} z^{k} \in F_{p, n}(a, c, \lambda, A, B)
\end{aligned}
$$

if and only if

$$
\left(\frac{1-B}{A-B}\right) \sum_{k=p+n}^{\infty} \frac{(p+(k-p) \lambda)(a)_{k-p}}{p(c)_{k-p}} a_{k, 1} a_{k, 2} \cdots a_{k, m} \leq 1 .
$$

To prove the result of Theorem 8, it follows from (27) and (29) that we need to find the smallest $A$ such that

$$
\begin{aligned}
& \frac{(p+(k-p) \lambda)(a)_{k-p}(1-B)}{p(c)_{k-p}(A-B)} \\
& \leq\left(\frac{(p+(k-p) \lambda)(a)_{k-p}}{p(c)_{k-p}}\right) \prod_{j=1}^{m}\left(\frac{1-B_{j}}{A_{j}-B_{j}}\right) \\
& \quad(k \geq p+n ; m \geq 2) ;
\end{aligned}
$$

that is, that

$$
\begin{aligned}
A \geq & B+(1-B)\left(\frac{p(c)_{k-p}}{(p+(k-p) \lambda)(a)_{k-p}}\right)^{m-1} \\
& \times \prod_{j=1}^{m}\left(\frac{A_{j}-B_{j}}{1-B_{j}}\right) \quad(k \geq p+n ; m \geq 2) .
\end{aligned}
$$

Since

$$
\frac{(c)_{k+1-p}}{(a)_{k+1-p}} \leq \frac{(c)_{k-p}}{(a)_{k-p}} \quad(k \geq p+n ; a \geq c>0)
$$

we see that the right-hand side of (31) is a decreasing function of $k$. Consequently, taking $k=p+n$ in (31), we have $h(z) \in$ $F_{p, n}(a, c, \lambda, A(B), B)$, where $A(B)$ is given by $(24)$. by

Furthermore, by considering the functions $f_{j}(z)$ defined

$$
\begin{array}{r}
f_{j}(z)=z^{p}-\frac{p(c)_{n}\left(A_{j}-B_{j}\right)}{(p+n \lambda)(a)_{n}\left(1-B_{j}\right)} z^{p+n} \\
(j=1,2, \ldots, m),
\end{array}
$$

we have $f_{j}(z) \in F_{p, n}\left(a, c, \lambda, A_{j}, B_{j}\right)$ and

$$
\begin{aligned}
& \left(f_{1} \cdot f_{2} \cdot \ldots \cdot f_{m}\right)(z) \\
& \quad=z^{p}-\left(\frac{p(c)_{n}}{(p+n \lambda)(a)_{n}}\right)^{m} \prod_{j=1}^{m}\left(\frac{A_{j}-B_{j}}{1-B_{j}}\right) z^{p+n} .
\end{aligned}
$$

Noting that

$$
\begin{aligned}
(p+n \lambda) & \frac{(a)_{n}}{(c)_{n}}\left(\frac{p(c)_{n}}{(p+n \lambda)(a)_{n}}\right) \prod_{j=1}^{m}\left(\frac{A_{j}-B_{j}}{1-B_{j}}\right) \\
& =p \frac{A(B)-B}{1-B}
\end{aligned}
$$

we conclude that $A(B)$ cannot be decreased for each $B$.

By using Lemma 4 instead of Lemma 3, the following theorem can be proved on the lines of the proof of Theorem 8 . We omit the details involved.

Theorem 9. Let $f_{j}(z) \in G_{p, n}\left(a, c, \lambda, A_{j}, B_{j}\right)(j=1,2, \ldots, m)$ and $a \geq c>0$. Then

$$
\left(f_{1} \cdot f_{2} \cdot \ldots \cdot f_{m}\right)(z) \in G_{p, n}(a, c, \lambda, A(B), B),
$$

where $m \geq 2$ and

$$
\begin{aligned}
A(B)= & B+(1-B)\left(\frac{p^{2}(c)_{n}}{(p+n)(p+n \lambda)(a)_{n}}\right)^{m-1} \\
& \times \prod_{j=1}^{m}\left(\frac{A_{j}-B_{j}}{1-B_{j}}\right) \in(B, 1) .
\end{aligned}
$$


The result is sharp for the functions $f_{j}(z)$ defined by

$$
\begin{aligned}
f_{j}(z) & =z^{p}-\frac{p^{2}(c)_{n}\left(A_{j}-B_{j}\right)}{(p+n)(p+n \lambda)(a)_{n}\left(1-B_{j}\right)} z^{p+n} \\
& \in G_{p, n}\left(a, c, \lambda, A_{j}, B_{j}\right) \quad(j=1,2, \ldots, m) .
\end{aligned}
$$

Theorem 10. Let $f_{j}(z) \in F_{p, n}\left(a, c, \lambda, A_{j}, B_{j}\right)(j=1,2, \ldots, m)$ with $a \geq c>0$ and $\lambda \geq 1$. Then

$$
\left(f_{1} \cdot f_{2} \cdot \ldots \cdot f_{m}\right)(z) \in G_{p, n}(a, c, \lambda, A(B), B),
$$

where $m \geq 2$ and

$$
\begin{aligned}
A(B)= & B+(1-B)\left(\frac{p+n}{p}\right)\left(\frac{p(c)_{n}}{(p+n \lambda)(a)_{n}}\right)^{m-1} \\
& \times \prod_{j=1}^{m}\left(\frac{A_{j}-B_{j}}{1-B_{j}}\right) .
\end{aligned}
$$

The result is sharp for the functions $f_{j}(z)(j=1,2, \ldots, m)$ defined by (33).

Proof. Obviously, $B<A(B) \leq 1$ for $m \geq 2, a \geq c>0$, and $\lambda \geq 1$. By applying Lemma 4 , we know that

$$
\begin{aligned}
& \left(f_{1} \cdot f_{2} \cdot \ldots \cdot f_{m}\right)(z) \\
& \quad=z^{p}-\sum_{k=p+n}^{\infty} a_{k, 1} a_{k, 2} \cdots a_{k, m} z^{k} \in G_{p, n}(a, c, \lambda, A, B),
\end{aligned}
$$

if and only if

$$
\left(\frac{1-B}{A-B}\right) \sum_{k=p+n}^{\infty} \frac{k(p+(k-p) \lambda)(a)_{k-p}}{p^{2}(c)_{k-p}} a_{k, 1} a_{k, 2} \cdots a_{k, m} \leq 1 .
$$

Proceeding as in the proof of Theorem 8 , we need to find the smallest $A$ such that

$$
\begin{aligned}
A \geq & B+(1-B) \frac{k}{p}\left(\frac{p(c)_{k-p}}{(p+(k-p) \lambda)(a)_{k-p}}\right)^{m-1} \\
& \times \prod_{j=1}^{m}\left(\frac{A_{j}-B_{j}}{1-B_{j}}\right) \quad(k \geq p+n) .
\end{aligned}
$$

Defining the function $\varphi(x)$ by

$$
\varphi(x)=\frac{x}{(p+(x-p) \lambda)^{m-1}} \quad(x \geq p+n),
$$

we see that

$$
\varphi^{\prime}(x)=\frac{p(1-\lambda)-(m-2) \lambda x}{(p+(x-p) \lambda)^{m}} \leq 0 \quad(x \geq p+n),
$$

for $m \geq 2$ and $\lambda \geq 1$. Hence, the right-hand side of (43) is a decreasing function of $k$. Thus, we arrive at $\left(f_{1} \cdot f_{2} \cdot \ldots \cdot f_{m}\right)(z) \in$ $G_{p, n}(a, c, \lambda, A(B), B)$, where $A(B)$ is given by $(40)$.

Sharpness can be verified easily.
By putting

$$
\begin{gathered}
a=c=1, \quad A(B)=1-\frac{2 \alpha}{p}, \quad B=-1, \\
A_{j}=1-\frac{2 \alpha_{j}}{p}, \quad B_{j}=-1 \quad(j=1,2, \ldots, m) .
\end{gathered}
$$

Theorem 10 reduces to the following.

Corollary 11. Let $f_{j}(z) \in F_{p}\left(n, \lambda, \alpha_{j}\right)(j=1,2, \ldots, m)$. Then $\left(f_{1} \cdot f_{2} \cdot \ldots \cdot f_{m}\right)(z) \in G_{p}(n, \lambda, \alpha)$, where $m \geq 2, \lambda \geq 1$, and

$$
\alpha=p-\frac{p+n}{p(p+n \lambda)^{m-1}} \prod_{j=1}^{m}\left(p-\alpha_{j}\right) .
$$

The result is sharp for the functions

$$
\begin{array}{r}
f_{j}(z)=z^{p}-\frac{p-\alpha_{j}}{p+n \lambda} z^{p+n} \in F_{p}\left(n, \lambda, \alpha_{j}\right) \\
(j=1,2, \ldots, m) .
\end{array}
$$

Theorem 12. Let $f_{j}(z) \in F_{p, n}\left(a, c, \lambda, A_{j}, B_{j}\right)(j=1,2, \ldots$, $m)$,

$$
\begin{gathered}
g_{j}(z) \in F_{p, n}\left(a, c, \lambda, C_{j}, D_{j}\right), \\
\left(-1 \leq D_{j} \leq 0 ; D_{j}<C_{j} \leq 1 ; j=1,2, \ldots, m\right)
\end{gathered}
$$

and $a \geq c>0$. Then one has

(i) $\sum_{j=1}^{m}\left(f_{j} \cdot g_{j}\right)(z)-(m-1) z^{p} \in F_{p, n}(a, c, \lambda, A(B), B)$, where

$$
\begin{gathered}
A(B)=B+(1-B) \frac{m p(c)_{n}\left(A_{0}-B_{0}\right)\left(C_{0}-D_{0}\right)}{(p+n \lambda)(a)_{n}\left(1-B_{0}\right)\left(1-D_{0}\right)}, \\
A_{0}=\max _{1 \leq j \leq m}\left\{A_{j}\right\}, \quad B_{0}=\min _{1 \leq j \leq m}\left\{B_{j}\right\}, \\
C_{0}=\max _{1 \leq j \leq m}\left\{C_{j}\right\}, \quad D_{0}=\min _{1 \leq j \leq m}\left\{D_{j}\right\}, \\
\text { provided that } A(B) \leq 1 .
\end{gathered}
$$

(ii) $(1 / m) \sum_{j=1}^{m}\left(f_{j} \cdot g_{j}\right)(z) \in F_{p, n}(a, c, \lambda, \widetilde{A}(B), B)$, where

$$
\begin{aligned}
\widetilde{A}(B)= & B+(1-B) \\
& \times \frac{p(c)_{n}\left(A_{0}-B_{0}\right)\left(C_{0}-D_{0}\right)}{(p+n \lambda)(a)_{n}\left(1-B_{0}\right)\left(1-D_{0}\right)} \in(B, 1] .
\end{aligned}
$$

Proof. It is clear that $-1 \leq B_{0} \leq B_{j}<A_{j} \leq A_{0} \leq 1, B_{0} \leq$ $0,-1 \leq D_{0} \leq D_{j}<C_{j} \leq C_{0} \leq 1, D_{0} \leq 0$,

$$
\begin{aligned}
& \frac{1-B_{j}}{A_{j}-B_{j}} \geq \frac{1-B_{0}}{A_{0}-B_{0}}>0, \\
& \frac{1-D_{j}}{C_{j}-D_{j}} \geq \frac{1-D_{0}}{C_{0}-D_{0}}>0,
\end{aligned}
$$


for $j=1,2, \ldots, m$. Let

$$
\begin{array}{r}
f_{j}(z)=z^{p}-\sum_{k=p+n}^{\infty} a_{k, j} z^{k} \in F_{p, n}\left(a, c, \lambda, A_{j}, B_{j}\right) \\
g_{j}(z)=z^{p}-\sum_{k=p+n}^{\infty} b_{k, j} z^{k} \in F_{p, n}\left(a, c, \lambda, C_{j}, D_{j}\right), \\
\quad(j=1,2, \ldots, m) .
\end{array}
$$

Then Lemma 3 gives

$$
\begin{aligned}
\sum_{j=1}^{m}\left(f_{j} \cdot g_{j}\right)(z)-(m-1) z^{p} & \\
= & z^{p}-\sum_{k=p+n}^{\infty}\left(\sum_{j=1}^{m} a_{k, j} b_{k, j}\right) z^{k} \in F_{p, n}(a, c, \lambda, A, B),
\end{aligned}
$$

if and only if

$$
\left(\frac{1-B}{A-B}\right) \sum_{k=p+n}^{\infty}\left\{\left(\frac{(p+(k-p) \lambda)(a)_{k-p}}{p(c)_{k-p}}\right)\left(\sum_{j=1}^{m} a_{k, j} b_{k, j}\right)\right\}
$$

$$
\leq 1 \text {. }
$$

Also, by Lemma 3 and (52), we deduce that

$$
\begin{aligned}
& \left(\frac{1-B_{0}}{A_{0}-B_{0}}\right)\left(\frac{1-D_{0}}{C_{0}-D_{0}}\right) \\
& \quad \times \sum_{k=p+n}^{\infty}\left(\frac{(p+(k-p) \lambda)(a)_{k-p}}{p(c)_{k-p}}\right)^{2} a_{k, j} b_{k, j} \\
& \leq\left\{\left(\frac{1-B_{j}}{A_{j}-B_{j}}\right) \sum_{k=p+n}^{\infty} \frac{(p+(k-p) \lambda)(a)_{k-p}}{p(c)_{k-p}} a_{k, j}\right\} \\
& \quad \times\left\{\left(\frac{1-D_{j}}{C_{j}-D_{j}}\right) \sum_{k=p+n}^{\infty} \frac{(p+(k-p) \lambda)(a)_{k-p}}{p(c)_{k-p}} b_{k, j}\right\} \\
& \leq 1 \quad(j=1,2, \ldots, m),
\end{aligned}
$$

and hence

$$
\begin{aligned}
& \frac{1}{m}\left(\frac{1-B_{0}}{A_{0}-B_{0}}\right)\left(\frac{1-D_{0}}{C_{0}-D_{0}}\right) \\
& \quad \times \sum_{k=p+n}^{\infty}\left\{\left(\frac{(p+(k-p) \lambda)(a)_{k-p}}{p(c)_{k-p}}\right)^{2}\left(\sum_{j=1}^{m} a_{k, j} b_{k, j}\right)\right\}
\end{aligned}
$$

$\leq 1$.
To prove Theorem 12(i), it follows from (55) and (57) that we need to find the smallest $A$ such that

$$
\begin{aligned}
A \geq & B+(1-B)\left(\frac{m p(c)_{k-p}}{(p+(k-p) \lambda)(a)_{k-p}}\right) \\
& \times\left(\frac{A_{0}-B_{0}}{1-B_{0}}\right)\left(\frac{C_{0}-D_{0}}{1-D_{0}}\right) \quad(k \geq p+n),
\end{aligned}
$$

for $a \geq c>0$. This leads to the assertion of Theorem 12(i). Analogously, we can prove Theorem 12(ii).

In the special case when

$$
\begin{gathered}
a=c=1, \quad A(B)=1-\frac{2 \alpha}{p}, \quad \widetilde{A}(B)=1-\frac{2 \widetilde{\alpha}}{p}, \\
B=-1, \quad A_{j}=C_{j}=1-\frac{2 \alpha_{j}}{p}, \\
B_{j}=D_{j}=-1 \quad(j=1,2, \ldots, m),
\end{gathered}
$$

Theorem 12 reduces to the following.

Corollary 13. Let $f_{j}(z) \in F_{p}\left(n, \lambda, \alpha_{j}\right)(j=1,2, \ldots, m)$. Then

(i) $\sum_{j=1}^{m}\left(f_{j} \cdot f_{j}\right)(z)-(m-1) z^{p} \in F_{p}(n, \lambda, \alpha)$, where

$$
\alpha=p-\frac{m}{p+n \lambda}\left(p-\min _{1 \leq j \leq m}\left\{\alpha_{j}\right\}\right)^{2},
$$

provided that $\alpha \geq 0$.

(ii) $(1 / m) \sum_{j=1}^{m}\left(f_{j} \cdot f_{j}\right)(z) \in F_{p}(n, \lambda, \widetilde{\alpha})$, where

$$
\widetilde{\alpha}=p-\frac{1}{p+n \lambda}\left(p-\min _{1 \leq j \leq m}\left\{\alpha_{j}\right\}\right)^{2} \in[0, p) .
$$

Replacing Lemma 3 by Lemma 4 in the proof of Theorem 12, one can prove the following.

Theorem 14. Let $f_{j}(z) \in G_{p, n}\left(a, c, \lambda, A_{j}, B_{j}\right)(j=1,2, \ldots$, $m)$,

$$
\begin{gathered}
g_{j}(z) \in G_{p, n}\left(a, c, \lambda, C_{j}, D_{j}\right), \\
\left(-1 \leq D_{j} \leq 0 ; D_{j}<C_{j} \leq 1 ; j=1,2, \ldots, m\right)
\end{gathered}
$$

and $a \geq c>0$. Then

(i) $\sum_{j=1}^{m}\left(f_{j} \cdot g_{j}\right)(z)-(m-1) z^{p} \in G_{p, n}(a, c, \lambda, A(B), B)$, where

$$
A(B)=B+(1-B) \frac{m p^{2}(c)_{n}\left(A_{0}-B_{0}\right)\left(C_{0}-D_{0}\right)}{(p+n)(p+n \lambda)(a)_{n}\left(1-B_{0}\right)\left(1-D_{0}\right)},
$$

and $A_{0}, B_{0}, C_{0}$, and $D_{0}$ are given as in Theorem 12, provided that $A(B) \leq 1$. 
(ii) $(1 / m) \sum_{j=1}^{m}\left(f_{j} \cdot g_{j}\right)(z) \in G_{p, n}(a, c, \lambda, \widetilde{A}(B), B)$, where

$$
\begin{aligned}
\widetilde{A}(B)= & B+(1-B) \\
& \times \frac{p^{2}(c)_{n}\left(A_{0}-B_{0}\right)\left(C_{0}-D_{0}\right)}{(n+p)(p+n \lambda)(a)_{n}\left(1-B_{0}\right)\left(1-D_{0}\right)} \in(B, 1) .
\end{aligned}
$$

As a special case of Theorem 14, one has the following.

Corollary 15. Let $f_{j}(z) \in G_{p}\left(n, \lambda, \alpha_{j}\right)(j=1,2, \ldots, m)$. Then

(i) $\sum_{j=1}^{m}\left(f_{j} \cdot f_{j}\right)(z)-(m-1) z^{p} \in G_{p}(n, \lambda, \alpha)$, where

$$
\alpha=p-\frac{m p}{(p+n)(p+n \lambda)}\left(p-\min _{1 \leq j \leq m}\left\{\alpha_{j}\right\}\right)^{2},
$$

provided that $\alpha \geq 0$.

(ii) $(1 / m) \sum_{j=1}^{m}\left(f_{j} \cdot f_{j}\right)(z) \in G_{p}(n, \lambda, \widetilde{\alpha})$, where

$$
\widetilde{\alpha}=p-\frac{p}{(p+n)(p+n \lambda)}\left(p-\min _{1 \leq j \leq m}\left\{\alpha_{j}\right\}\right)^{2} \in(0, p) \text {. }
$$

\section{Integral Operator}

Theorem 16. Let

$$
\begin{array}{r}
0 \leq \lambda_{1} \leq \lambda_{0}, \quad 0<c_{0} \leq a_{0}, \quad 0<a_{1} \leq c_{1} \\
\left(\text { or } 0<a_{1} \leq a_{0} \text { and } 0<c_{0} \leq c_{1}\right) .
\end{array}
$$

If $f(z) \in F_{p, n}\left(a_{0}, c_{0}, \lambda_{0}, A, B\right)$, then the function $I(z)$ defined by

$$
I(z)=\frac{\mu+p}{z^{\mu}} \int_{0}^{z} t^{\mu-1} f(t) d t \quad(\mu>-p)
$$

belongs to $F_{p, n}\left(a_{1}, c_{1}, \lambda_{1}, C(D), D\right)$, where $-1 \leq D \leq 0$ and

$$
\begin{aligned}
C(D)= & D+(1-D) \\
& \times \frac{\left(p+n \lambda_{1}\right)\left(a_{1}\right)_{n}\left(c_{0}\right)_{n}(\mu+p)(A-B)}{\left(p+n \lambda_{0}\right)\left(c_{1}\right)_{n}\left(a_{0}\right)_{n}(\mu+p+n)(1-B)} .
\end{aligned}
$$

The result is sharp; that is, the number $C(D)$ cannot be decreased for each $D$.

Proof. Note that $D<C(D)<1$. For

$$
f(z)=z^{p}-\sum_{k=p+n}^{\infty} a_{k} z^{k} \in F_{p, n}\left(a_{0}, c_{0}, \lambda_{0}, A, B\right),
$$

it follows from (68) that

$$
I(z)=z^{p}-\sum_{k=p+n}^{\infty} \frac{\mu+p}{\mu+k} a_{k} z^{k} \quad(z \in U ; \mu>-p) .
$$

To prove the result of Theorem 16, we need to find the smallest $C$ such that

$$
\begin{array}{r}
C \geq D+(1-D) \\
\times \frac{\left(p+(k-p) \lambda_{1}\right)\left(a_{1}\right)_{k-p}\left(c_{0}\right)_{k-p}(\mu+p)(A-B)}{\left(p+(k-p) \lambda_{0}\right)\left(c_{1}\right)_{k-p}\left(a_{0}\right)_{k-p}(\mu+k)(1-B)} \\
(k \geq p+n),
\end{array}
$$

where we have used Lemma 3. In view of (67), it is easy to know that the right-hand side of (72) is a decreasing function of $k$. Therefore, we conclude that

$$
I(z) \in F_{p, n}\left(a_{1}, c_{1}, \lambda_{1}, C(D), D\right),
$$

where $C(D)$ is given by (69).

Furthermore, it can easily be verified that the result is sharp, with the extremal function

$$
\begin{aligned}
f(z)= & z^{p}-\frac{p\left(c_{0}\right)_{n}(A-B)}{\left(p+n \lambda_{0}\right)\left(a_{0}\right)_{n}(1-B)} \\
& \times z^{p+n} \in F_{p, n}\left(a_{0}, c_{0}, \lambda_{0}, A, B\right) .
\end{aligned}
$$

With the aid of Lemma 4 (instead of Lemma 3) and using the same steps as in the proof of Theorem 16, we can prove the following.

Theorem 17. Let (67) in Theorem 16 be satisfied. If $f(z) \in$ $G_{p, n}\left(a_{0}, c_{0}, \lambda_{0}, A, B\right)$, then the function $I(z)$ defined by (68) belongs to $G_{p, n}\left(a_{1}, c_{1}, \lambda_{1}, C(D), D\right)$, where $C(D)(-1 \leq D \leq 0)$ is the same as in Theorem 16. The result is sharp for the function

$$
\begin{aligned}
f(z)= & z^{p}-\frac{p^{2}\left(c_{0}\right)_{n}(A-B)}{(p+n)\left(p+n \lambda_{0}\right)\left(a_{0}\right)_{n}(1-B)} \\
& \times z^{p+n} \in G_{p, n}\left(a_{0}, c_{0}, \lambda_{0}, A, B\right) .
\end{aligned}
$$

If we let

$$
\begin{gathered}
a_{0}=c_{0}=a_{1}=c_{1}=1, \quad \lambda_{0}=\lambda_{1}=\lambda \geq 0, \\
A=1-\frac{2 \alpha}{p}, \quad B=D=-1, \quad C(D)=1-\frac{2 \beta(\alpha)}{p},
\end{gathered}
$$

then Theorem 17 yields the following.

Corollary 18. Let $f(z) \in G_{p}(n, \lambda, \alpha)$. Then the function $I(z)$ defined by (68) belongs to $G_{p}(n, \lambda, \beta(\alpha))$, where

$$
\beta(\alpha)=\frac{\alpha(\mu+p)+n p}{\mu+p+n} .
$$

The number $\beta(\alpha)$ cannot be increased for each $\alpha \in[0, p)$. 


\section{Partial Sums}

In this section, we let $f(z) \in T_{p, n}$ be given by (6) and define the partial sums $s_{1}(z)$ and $s_{m}(z)$ by

$$
s_{1}(z)=z^{p}, \quad s_{m}(z)=z^{p}-\sum_{k=p+n}^{p+n+m-2} a_{k} z^{k},
$$

$$
(m \in N \backslash\{1\}) \text {. }
$$

Also we make the notation simple by writing

$$
\begin{array}{r}
\beta_{k}=\frac{(p+(k-p) \lambda)(a)_{k-p}(1-B)}{p(c)_{k-p}(A-B)} \\
(k=p+n, p+n+1, \ldots) .
\end{array}
$$

Theorem 19. Let $f(z) \in F_{p, n}(a, c, \lambda, A, B)$ and $a \geq c>0$. Then for $z \in U$, one has the following.

$$
\begin{aligned}
& \operatorname{Re} \frac{f(z)}{s_{m}(z)}>1-\frac{1}{\beta_{p+n+m-1}} \quad(m \in N), \\
& \operatorname{Re} \frac{s_{m}(z)}{f(z)}>\frac{\beta_{p+n+m-1}}{1+\beta_{p+n+m-1}} \quad(m \in N) .
\end{aligned}
$$

The results are sharp for each $m \in N$.

Proof. Let $a \geq c>0$ and $\beta_{k}$ be given by (79). Then $\beta_{k+1} \geq$ $\beta_{k} \geq 1$ for $k \geq p+n$, and so it follows from Lemma 3 that

$$
\begin{gathered}
\sum_{k=p+n}^{p+n+m-2} a_{k}+\beta_{p+n+m-1} \cdot \sum_{k=p+n+m-1}^{\infty} a_{k} \\
\leq \sum_{k=p+n}^{\infty} \beta_{k} a_{k} \leq 1 \quad(m \geq 2),
\end{gathered}
$$

for $f(z) \in F_{p, n}(a, c, \lambda, A, B)$.

If we put

$$
\begin{array}{r}
p_{1}(z)=1+\beta_{p+n+m-1}\left(\frac{f(z)}{s_{m}(z)}-1\right) \\
=1-\frac{\beta_{p+n+m-1} \cdot \sum_{k=p+n+m-1}^{\infty} a_{k} z^{k-p}}{1-\sum_{k=p+n}^{p+n+m-2} a_{k} z^{k-p}} \\
(z \in U ; m \geq 2),
\end{array}
$$

then $p(0)=1$ and

$$
\begin{aligned}
& \left|\frac{p_{1}(z)-1}{p_{1}(z)+1}\right| \\
& \quad \leq \frac{\beta_{p+n+m-1} \cdot \sum_{k=p+n+m-1}^{\infty} a_{k}}{2-2 \sum_{k=p+n}^{p+n+m-2} a_{k}-\beta_{p+n+m-1} \cdot \sum_{k=p+n+m-1}^{\infty} a_{k}} \\
& \quad \leq 1 \quad(z \in U ; m \geq 2)
\end{aligned}
$$

because of (82). Hence, we have $\operatorname{Re} p_{1}(z)>0$ for $z \in U$, which implies that (80) holds true for $m \geq 2$.

Similarly, by setting

$$
p_{2}(z)=\left(1+\beta_{p+n+m-1}\right) \frac{s_{m}(z)}{f(z)}-\beta_{p+n+m-1} \quad(z \in U)
$$

it follows from (82) that

$$
\begin{aligned}
& \left|\frac{p_{2}(z)-1}{p_{2}(z)+1}\right| \\
& \quad \leq \frac{\left(1+\beta_{p+n+m-1}\right) \cdot \sum_{k=p+n+m-1}^{\infty} a_{k}}{2-2 \sum_{k=p+n}^{p+n+m-2} a_{k}-\left(\beta_{p+n+m-1}-1\right) \cdot \sum_{k=p+n+m-1}^{\infty} a_{k}} \\
& \quad \leq 1 \quad(z \in U ; m \geq 2) .
\end{aligned}
$$

Therefore, we see that $\operatorname{Re} p_{2}(z)>0$ for $z \in U$, that is, that (81) holds for $m \geq 2$.

For $m=1$, replacing (82) by

$$
\beta_{p+n} \sum_{k=p+n}^{\infty} a_{k} \leq \sum_{k=p+n}^{\infty} \beta_{k} a_{k} \leq 1
$$

and proceeding as the above, we know that (80) and (81) are also true.

Furthermore, by taking the function

$$
f(z)=z^{p}-\frac{z^{p+n+m-1}}{\beta_{p+n+m-1}},
$$

we find that $s_{m}(z)=z^{p}$,

$$
\begin{gathered}
\operatorname{Re} \frac{f(z)}{s_{m}(z)} \longrightarrow 1-\frac{1}{\beta_{p+n+m-1}} \quad \text { as } z \longrightarrow 1, \\
\operatorname{Re} \frac{s_{m}(z)}{f(z)} \longrightarrow \frac{\beta_{p+n+m-1}}{1+\beta_{p+n+m-1}} \text { as } z \longrightarrow \exp \left(\frac{\pi i}{n+m-1}\right) .
\end{gathered}
$$

The proof of Theorem 19 is thus completed.

By virtue of Theorem 19 and Definition 2, we easily have the following.

Corollary 20. Let $f(z) \in G_{p, n}(a, c, \lambda, A, B)$ and $a \geq c>0$. Then we have

$$
\begin{aligned}
& \operatorname{Re} \frac{f^{\prime}(z)}{s_{m}^{\prime}(z)}>1-\frac{1}{\beta_{p+n+m-1}} \quad(z \in U ; m \in N), \\
& \operatorname{Re} \frac{s_{m}^{\prime}(z)}{f^{\prime}(z)}>\frac{\beta_{p+n+m-1}}{1+\beta_{p+n+m-1}} \quad(z \in U ; m \in N) .
\end{aligned}
$$

The results are sharp for each $m \in N$. 
Theorem 21. Let $f(z) \in F_{p, n}(a, c, \lambda, A, B)$ with $a \geq c>0$ and $\lambda \geq 1$. Then one has

$$
\begin{gathered}
\operatorname{Re} \frac{f^{\prime}(z)}{s_{m}^{\prime}(z)}>1-\frac{p+n+m-1}{p \beta_{p+n+m-1}} \quad(z \in U ; m \in N), \\
\operatorname{Re} \frac{s_{m}^{\prime}(z)}{f^{\prime}(z)}>\frac{p \beta_{p+n+m-1}}{p+n+m-1+p \beta_{p+n+m-1}} \quad(z \in U ; m \in N) .
\end{gathered}
$$

The results are sharp for each $m \in N$.

Proof. Let $a \geq c>0, \lambda \geq 1$ and $\beta_{k}$ be given by (79). Then it is easy to verify that

$$
\frac{\beta_{k+1}}{k+1} \geq \frac{\beta_{k}}{k} \geq \frac{1}{p} \quad(k \geq p+n)
$$

and hence we deduce from Lemma 3 that

$$
\begin{aligned}
& \sum_{k=p+n}^{p+n+m-2} \frac{k}{p} a_{k}+\frac{\beta_{p+n+m-1}}{p+n+m-1} \cdot \sum_{k=p+n+m-1}^{\infty} k a_{k} \\
& \leq \sum_{k=p+n}^{\infty} \beta_{k} a_{k} \leq 1 \quad(m \geq 2),
\end{aligned}
$$

for $f(z) \in F_{p, n}(a, c, \lambda, A, B)$.

Defining the function $q_{1}(z)$ by

$$
q_{1}(z)=1+\frac{p \beta_{p+n+m-1}}{p+n+m-1}\left(\frac{f^{\prime}(z)}{s_{m}^{\prime}(z)}-1\right) \quad(z \in U),
$$

it follows from (94) that

$$
\begin{aligned}
& \left|\frac{q_{1}(z)-1}{q_{1}(z)+1}\right| \\
& \leq\left(\frac{p \beta_{p+n+m-1}}{p+n+m-1} \cdot \sum_{k=p+n+m-1}^{\infty} k a_{k}\right) \\
& \quad \times\left(2 p-2 \sum_{k=p+n}^{p+n+m-2} k a_{k}-\frac{p \beta_{p+n+m-1}}{p+n+m-1}\right. \\
& \left.\cdot \sum_{k=p+n+m-1}^{\infty} k a_{k}\right)^{-1} \\
& \leq 1 \quad(z \in U ; m \geq 2) .
\end{aligned}
$$

This leads to the inequality (91) for $m \geq 2$.

Similarly, for the function $q_{2}(z)$ defined by

$$
q_{2}(z)=\left(1+\frac{p \beta_{p+n+m-1}}{p+n+m-1}\right) \frac{s_{m}^{\prime}(z)}{f^{\prime}(z)}-\frac{p \beta_{p+n+m-1}}{p+n+m-1},
$$

we deduce from (94) that

$$
\begin{aligned}
& \left|\frac{q_{2}(z)-1}{q_{2}(z)+1}\right| \\
& \leq\left(\left(1+\frac{p \beta_{p+n+m-1}}{p+n+m-1}\right) \cdot \sum_{k=p+n+m-1}^{\infty} k a_{k}\right) \\
& \times\left(2 p-2 \sum_{k=p+n}^{p+n+m-2} k a_{k}-\left(\frac{p \beta_{p+n+m-1}}{p+n+m-1}-1\right)\right. \\
& \left.\cdot \sum_{k=p+n+m-1}^{\infty} k a_{k}\right)^{-1}
\end{aligned}
$$$$
\leq 1 \quad(z \in U ; m \geq 2) \text {. }
$$

This yields the inequality (92) for $m \geq 2$.

For $m=1$, replacing (94) by

$$
\frac{\beta_{p+n}}{p+n} \sum_{k=p+n}^{\infty} k a_{k} \leq \sum_{k=p+n}^{\infty} \beta_{k} a_{k} \leq 1,
$$

we know that (91) and (92) are also true.

Furthermore, the bounds in (91) and (92) are the best possible for the function $f(z)$ defined by (88).

Finally, Theorem 21 yields the following.

Corollary 22. Let $f(z) \in G_{p, n}(a, c, \lambda, A, B)$ with $a \geq c>0$ and $\lambda \geq 1$. Then

$$
\begin{aligned}
& \operatorname{Re} \frac{f^{\prime}(z)+z f^{\prime \prime}(z)}{s_{m}^{\prime}(z)+z s_{m}^{\prime \prime}(z)}>1-\frac{p+n+m-1}{p \beta_{p+n+m-1}}(z \in U ; m \in N), \\
& \operatorname{Re} \frac{s_{m}^{\prime}(z)+z s_{m}^{\prime \prime}(z)}{f^{\prime}(z)+z f^{\prime \prime}(z)}>\frac{p \beta_{p+n+m-1}}{p+n+m-1+p \beta_{p+n+m-1}} \\
&(z \in U ; m \in N) .
\end{aligned}
$$

The results are sharp for each $m \in N$.

\section{Conflict of Interests}

The authors declare that there is no conflict of interests regarding the publication of this paper.

\section{References}

[1] B. C. Carlson and D. B. Shaffer, "Starlike and prestarlike hypergeometric functions," SIAM Journal on Mathematical Analysis, vol. 15, no. 4, pp. 737-745, 1984.

[2] J. Dziok, "Classes of functions defined by certain differentialintegral operators," Journal of Computational and Applied Mathematics, vol. 105, no. 1-2, pp. 245-255, 1999. 
[3] J. Dziok and H. M. Srivastava, "Classes of analytic functions associated with the generalized hypergeometric function," Applied Mathematics and Computation, vol. 103, no. 1, pp. 1-13, 1999.

[4] S. K. Lee, S. Owa, and H. M. Srivastava, "Basic properties and characterizations of a certain class of analytic functions with negative coefficients," Utilitas Mathematica, vol. 36, pp. 121-128, 1989.

[5] M. K. Aouf and H. E. Darwish, "Basic properties and characterizations of a certain class of analytic functions with negative coefficients. II," Utilitas Mathematica, vol. 46, pp. 167-177, 1994.

[6] M. K. Aouf, H. M. Hossen, and H. M. Srivastava, "A certain subclass of analytic p-valent functions with negative coefficients," Demonstratio Mathematica, vol. 31, no. 3, pp. 595-608, 1998.

[7] T. Yaguchi, O. Kwon, N. E. Cho, and R. Yamakawa, "A generalization class of certain subclasses of $p$-valently analytic functions with negative coefficients," Research Institute for Mathematical Sciences Kokyuroku, no. 821, pp. 101-111, 1993.

[8] M. K. Aouf, "A subclass of analytic $p$-valent functions with negative coefficients. I," Utilitas Mathematica, vol. 46, pp. 219231, 1994.

[9] M. K. Aouf, "The quasi-Hadamard products of certain subclasses of analytic $p$-valent functions with negative coefficients," Applied Mathematics and Computation, vol. 187, no. 1, pp. 54-61, 2007.

[10] J. Dziok, "A unified class of analytic functions with fixed argument of coefficients," Acta Mathematica Scientia B, vol. 31, no. 4, pp. 1357-1366, 2011.

[11] J. Dziok, "On the convex combination of the Dziok-Srivastava operator," Applied Mathematics and Computation, vol. 188, no. 2, pp. 1214-1220, 2007. 


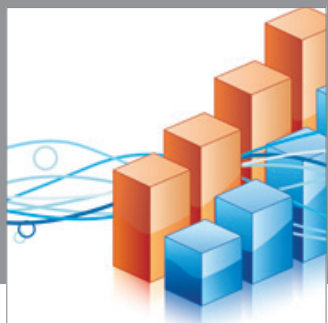

Advances in

Operations Research

mansans

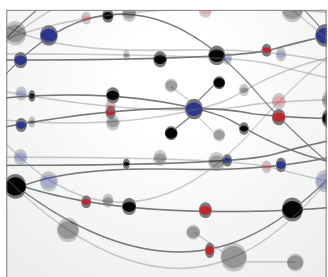

The Scientific World Journal
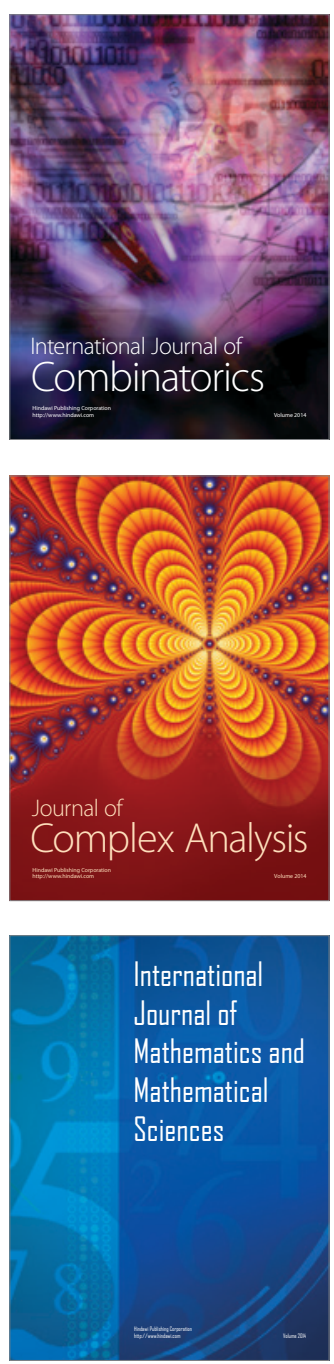
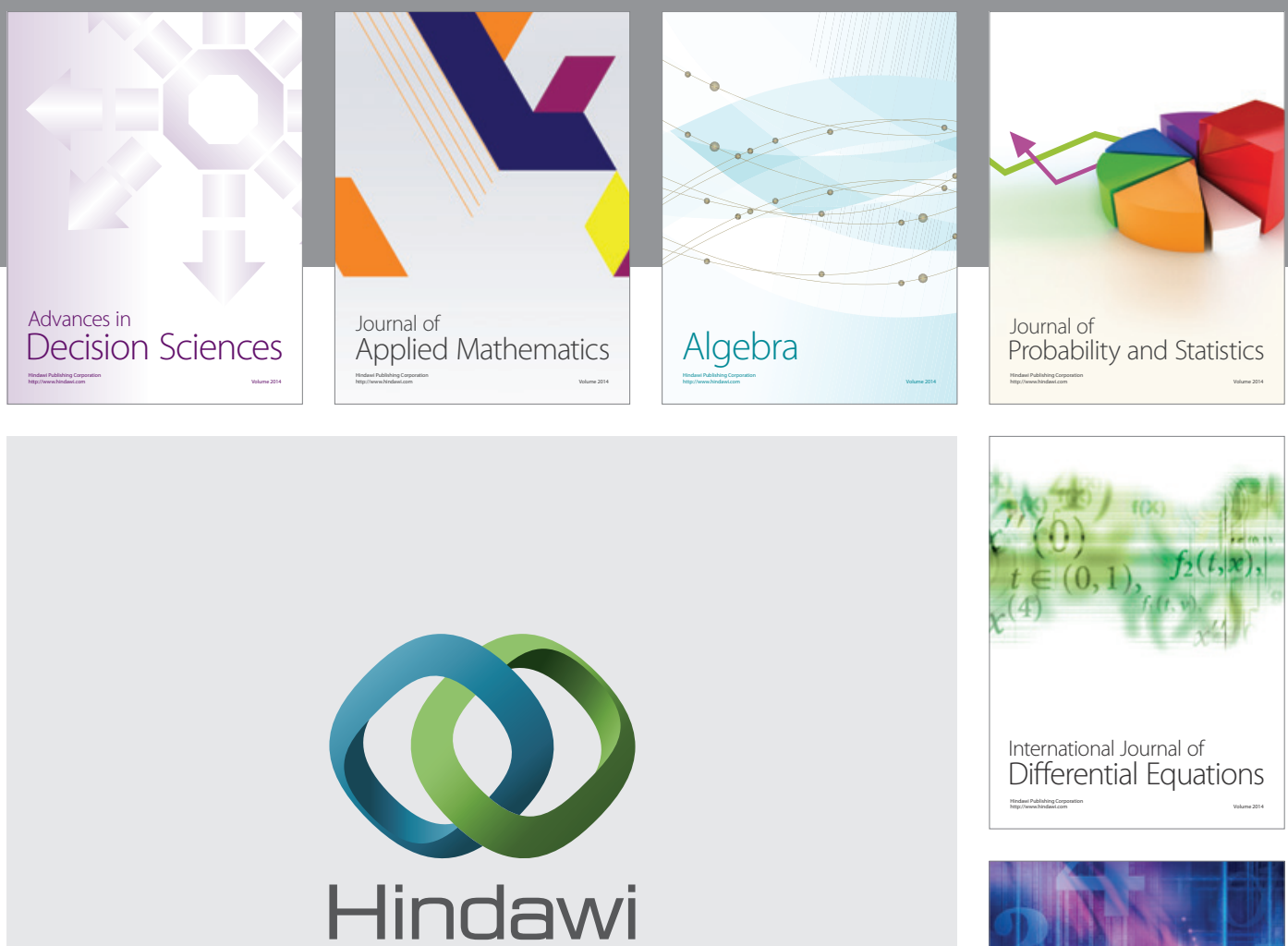

Submit your manuscripts at http://www.hindawi.com
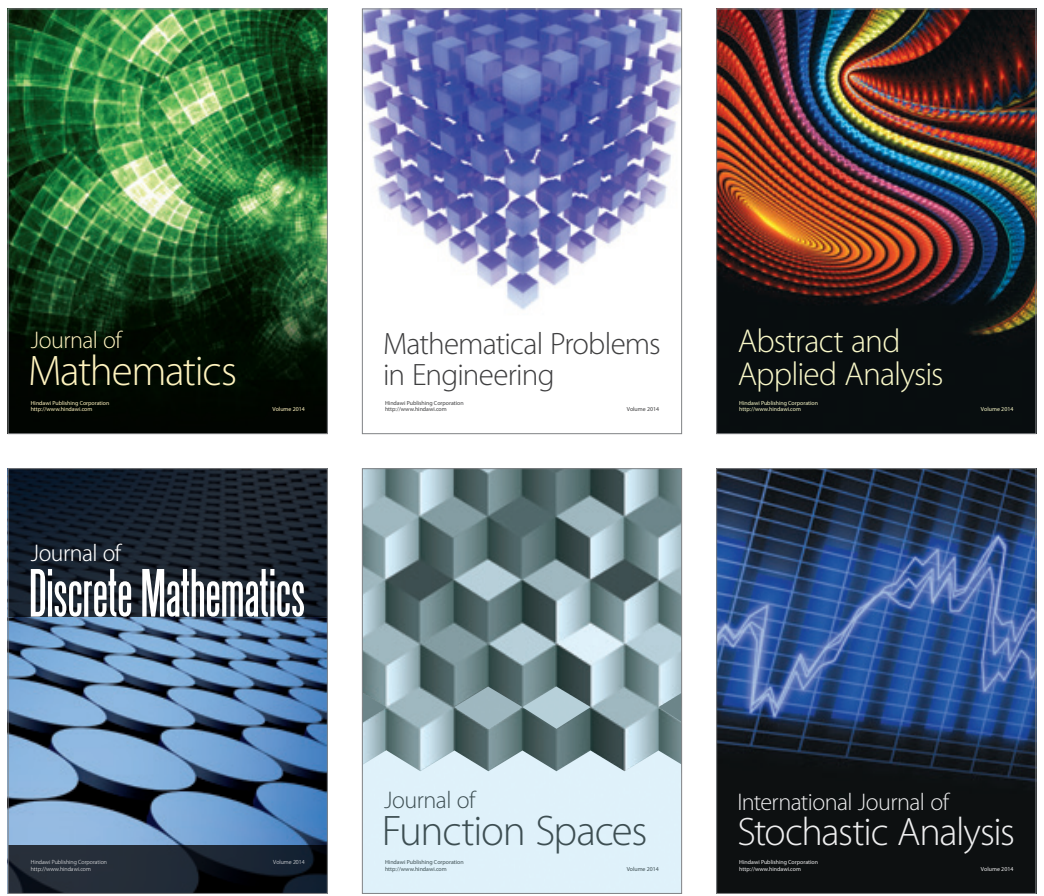

Journal of

Function Spaces

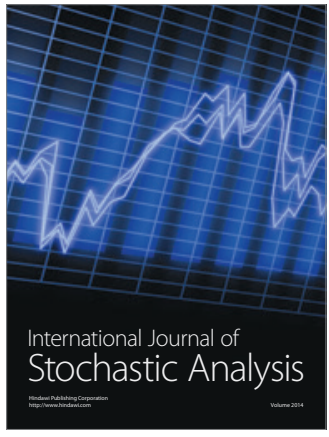

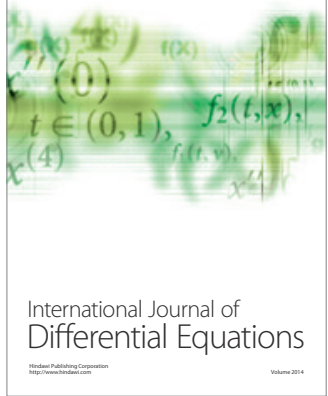
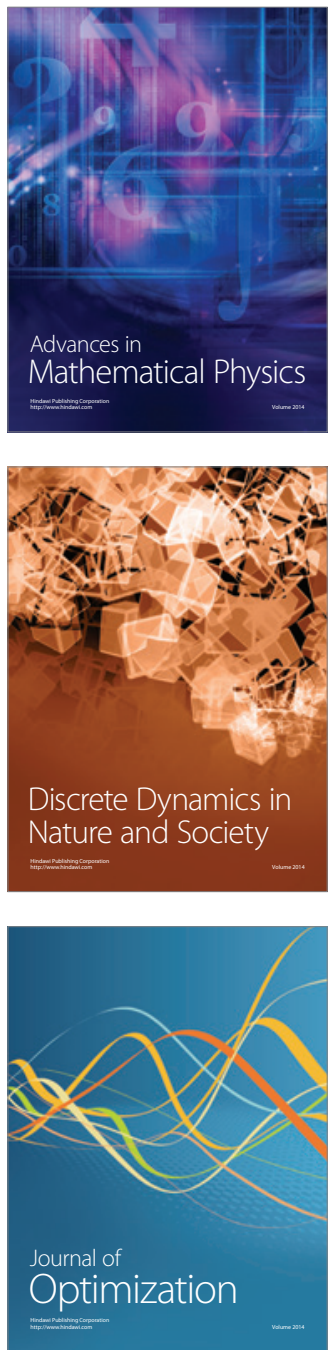